\title{
Robots: Automatización en Diseño y Construcción para la Enseñanza de Arquitectura
}

\author{
Robots: Automation in Design and Manufacturing for Teaching Architecture
}

\author{
Mauro Chiarella \\ Universidad Nacional del Litoral, Argentina \\ CONICET, Argentina \\ chiarell@fadu.unl.edu.ar \\ Luis Felipe González Böhme \\ Universidad Técnica Federico Santa María, Chile \\ luisfelipe.gonzalez@usm.cl
}

\author{
Cristian Calvo Barentin \\ Universidad Técnica Federico Santa María, Chile \\ cristian.calvo@usm.cl
}

\begin{abstract}
Industrial robots controlled by parametric design software and visual programming environments are gaining popularity in the research and use of non-conventional construction processes in architecture. Process automation which can be personalized through variable components promises to become an industry standard with similar cost structures to current pre-fabrication industrial processes. In order to incorporate competencies from non-serial variable architectural modular design, an initial teaching initiative (Advanced Architectural Design Studio - USM) was developed in Latin América. The strategy employed is based on incorporating concepts and instruments of Construction \& Design Automation for CAD/CAM processes with a Six Axis Robotic Arm (KUKA KR125/2).
\end{abstract}

Keywords: Robotic fabrication; Parametric modeling; Teaching architecture.

\section{Robots en Arquitectura}

Los Robots industriales controlados por software de diseño paramétrico y ambientes de programación visual están ganando popularidad en las investigaciones y uso de procesos constructivos no convencionales en Arquitectura. La automatización de procesos con posibilidad de personalización e individualización de componentes variables promete su utilización masiva a costos similares a la estandarización industrial de los modelos conocidos de prefabricación. Si bien esta afirmación no es verificable aún en todos los casos, se visualiza claramente su potencialidad a futuro y las actuales investigaciones trabajan con optimismo hacia ese sentido. Con el objetivo de incorporar competencias en el diseño modular no seriado de componentes arquitectónicos variables, se desarrolla una primera experiencia pedagógica (Taller Avanzado de Diseño Arquitectónico-USM) en Latinoamérica. La estrategia utilizada se sostiene en la incorporación incipiente de conceptos e instrumentos de Automatización en Construcción y Diseño para procesos CAD/CAM con Brazo Robótico de 6 ejes (KR125/2).

El amplio desarrollo de los sistemas de ideación digitales, en las últimas décadas, han terminado desafiando a los sistemas de producción análogos en poder concretar y materializar muchas de las ideas que se generan virtualmente. Los sistemas CAD-CAM han redefinidos las instancias de pre-figuración y representación de las disciplinas vinculadas al diseño. Es así como han condicionado y transformado procesos de fabricación y construcción, modificando su metodología operacional obligando a salir de lo estrictamente gráfico, ampliando horizontes (Chiarella, 2004). Estas tecnologías de fabricación digital se clasifican según los procedimientos empleados sobre las propiedades físicas y/o químicas de los materiales que manipulan: Procedimientos Generativos; Substractivos; Transformativos y de Unión de Componentes. Los brazos robóticos pueden realizar la mayoría de los procedimientos, dependiendo de la herramienta que sostengan en un extremo. Debido a su limitación de área de trabajo, una nueva modularidad de componentes se impone proponiendo formas de ensamblajes no seriadas. La misma no responde necesariamente a una rigurosidad cartesiana (como en los sistemas prefabricados convencionales) sino que se diseña estratégicamente con relación a una composición integral más compleja. La incorporación estratégica del cálculo matemático en las definiciones espaciales y geométricas basadas en el Modelado Paramétrico, ha sido determinante para estas configuraciones espaciales y morfológicas de los proyectos que asumen estos desafíos. La expresión material de estas configuraciones establece una importante vinculación entre la conceptualización formal, la operación geométrica digital, su elaboración material y revisión sensible, de manera análoga al proceso de diseño y fabricación utilizado. 


\section{Taller Avanzado de Diseño Arquitectónico-USM}

El Departamento de Arquitectura de la Universidad Técnica Federico Santa María se encuentra inserto dentro un campus universitario orientado a la formación de futuros ingenieros y a la investigación y desarrollo de conocimiento en ciencia y tecnología. Esto posibilita que la carrera de Arquitectura de esta Universidad cuente con una gran infraestructura para potenciar el desarrollo de sus estudiantes en distintas áreas relacionadas con Ciencia y Tecnología. Es por esto que el Área de profundización en Computación del Departamento de Arquitectura y el Centro Integrado de Manufactura y Automatización (CIMA) de la misma Universidad iniciaron un trabajo conjunto para explorar las distintas posibilidades en la utilización de robots industriales en procesos de fabricación digital. El Taller Avanzado de Diseño Arquitectónico, de cursado semestral, propuso en el primer semestre de 2013 un ejercicio de diseño de una torre de salvavidas prefabricable y desmontable, con criterios de emplazamientos para un sector predefinido de las costas marítimas chilenas. El ejercicio se inició con procesos tradicionales (análogos-digitales), induciendo desde los inicios una mirada estratégica en el control geométrico de la forma que pudiera contener componentes materiales prefabricados (estructurales y de cerramientos) de montaje en seco.

\section{Automatización en diseño y construcción}

La incorporación de conceptos e instrumentos de automatización para el diseño y la manufactura de componentes, se realizó paralelamente al avance del ejercicio de diseño. La construcción experimental de las primeras piezas y componentes posibles de ensamblar, se realizó en la segunda mitad del semestre.

Como primer acercamiento al tema se consultaron los trabajos de dos referentes europeos que ya cuentan con diversos trabajos en esta línea de investigación. El primero de ellos es el grupo de arquitectos suizos de la ETH Zürich, Fabio Gramazio y Matthias Kohler, quienes fueron pioneros en la utilización de estos brazos robóticos en procesos de fabricación digital. El segundo referente es el grupo austriaco "Association for Robots in Architecture" de la TU Wien liderado por Sigrid Brell-Cokcan y Johannes Braumann. Este grupo desarrollo un software que funciona sobre el ambiente de programación Grasshopper $^{\circledR}$ y el cual permite programar las secuencias de fresado de los brazos robotizados mediante diseño paramétrico. Esto posibilita que diseñadores que no manejan lenguajes de programación complejos puedan utilizar estas herramientas para explorar nuevas posibilidades en la fabricación digital.

\section{Ejercicios iniciales}

Los primeros intentos de programar una secuencia de movimiento para el robot Kuka KR6 se basaron en el tutorial disponible en la página web de la "Association for Robots in Architecture". Este tutorial explica paso a paso desde como instalar el plug-in KUKA|prc en la plataforma Grasshopper ${ }^{\circledR}$ hasta los conceptos geométricos básicos en la programación de trayectorias de movimiento del brazo robótico. Estos conceptos y su relación con las herramientas de programación presentes en el programa KUKA|prc y Grasshopper ${ }^{\circledR}$ son expuestos a través de un ejercicio virtual de cortes mediante movimientos simétricos y reiterativos. La configuración del tipo de herramienta y la posición del stock con respecto a la base de robot son algunas de las variables a considerar. El programa KUKA|prc tiene la posibilidad de traducir el programa construido paramétricamente en Grasshopper al lenguaje nativo de KUKA, el cual tiene la extensión .src y se puede cargar directamente en la CPU del robot. Otra posibilidad que ofrece el software KUKA|prc es la de poder simular los movimientos del robot a partir del programa generado $y$ exportarlo a un archivo con formato de video. Esto es muy útil cuando el programa contiene muchos movimientos y es imposible analizar la secuencia en detalle. Con el video se puede revisar la secuencia de movimiento completa a la velocidad necesaria para detectar posibles movimientos no deseados o giros que sobrepasan el rango máximo posible de cada uno de los ejes del robot.

\section{Pruebas geométricas}

Las primeras pruebas se basaron en la secuencia de movimientos documentada en el tutorial de la "Association for Robots in Architecture". Este archivo fue cargado en el brazo robot KUKA KR6 perteneciente al CIMA para poder observar la ejecución de la secuencia diseñada. Al no contar con ningún tipo de herramienta para acoplar al extremo del robot solo se ejecuto la secuencia de movimientos sin llegar a cortar ningún elemento de prueba. Las primeras pruebas fueron exitosas; por lo tanto se generaron dos secuencias de cortes diferentes para explorar las posibilidades de movimiento.

Cinta: El segundo código generado fue una cinta alabeada que cuenta con una curva base sobre la cual se mueve el extremo de la herramienta y una curva guía, que determina la dirección $(x, y, z)$ en el espacio que adquiere el eje de elongación de la herramienta en la medida que esta se mueve a través de la trayectoria.

Cilindro: El tercer ejercicio fue generar un código para comprobar que el brazo robótico es capaz de trazar trayectorias curvas generadas por vectores normales a una superficie determinada. Esta superficie generada es un cilindro cuyo eje de elongación es paralelo al eje $\mathrm{Y}$.

Montaje de herramienta: Para poder realizar pruebas de corte se cotizaron distintas herramientas de fresado, donde las características más importantes eran el precio, su calidad y la forma en cómo acoplarla al brazo robótico KUKA Kr 125/2. Se escogió una amoladora con características industriales de la marca alemana Metabo. Para acoplar la herramienta al brazo, se diseño un soporte basado en las indicaciones proporcionadas por Johannes Braumann de la "Association for Robots in Architecture". Este soporte fue mecanizado en Technyl por personal del laboratorio de materiales del Departamento de Arquitectura. 


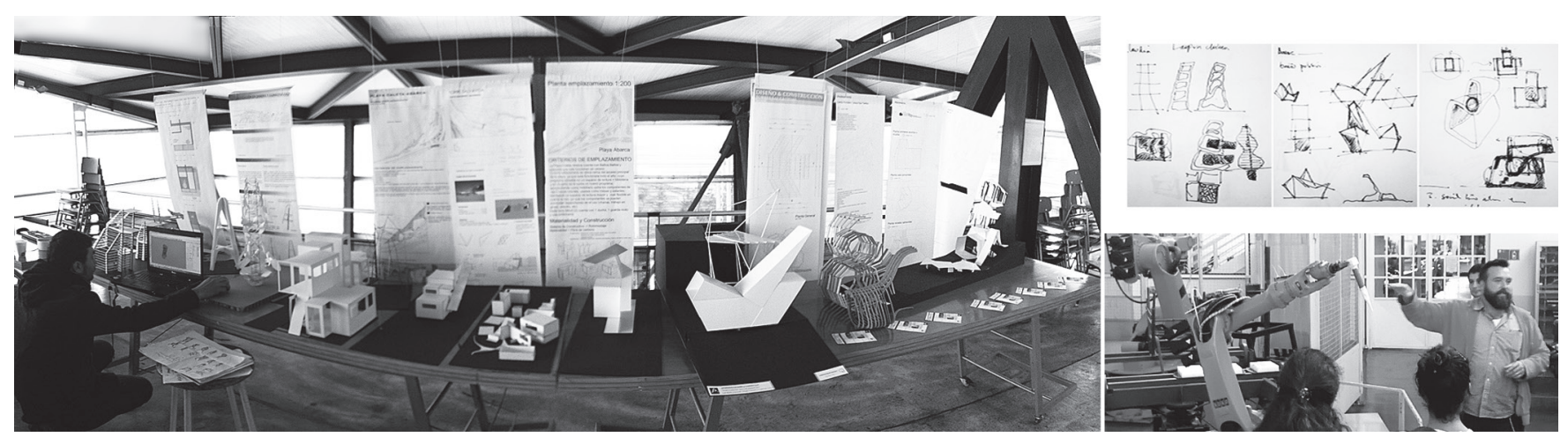

Figure 1: Taller Avanzado de Diseño Arquitectónico. ADC2013. USM

\section{Componentes Variables}

\section{Primeras pruebas: celosías-paneles}

Como ejercicio de taller se conformaron grupos de dos integrantes cada uno, para luego asignarles a cada uno, una tipología de celosía. La cual tenían que diseñar paramétricamente en Grasshopper y luego programar las secuencias de corte en KUKA|prc. Tres tipologías de celosías fueron seleccionadas por el equipo docente para ser asignadas dentro del ejercicio de taller. Estas están compuestas por polígonos, círculos o líneas y cuentan con tres niveles diferentes de densidad. Estas densidades responden a parámetros que controlan en distintos grados el paso de la luz y la privacidad de un espacio a configurar. Las primeras aproximaciones de los estudiantes no fueron del todo exitosas ya que muchos tuvieron problemas a la hora de programar las secuencias de fresado mediante KUKA / prc.

\section{Celosías 2.0}

Para lograr obtener resultados que pudiesen ser evaluados desde la posibilidad de utilizar el brazo robot KUKA, se seleccionaron los ejercicios que tuviesen factibilidad de ser fresados. Estos archivos fueron revisados y actualizados por el equipo docente con el fin de poder corregir errores en la programación para así poder fresarlos. Las experiencias de fresado asistido mediante un brazo robótico industrial fueron realizadas con un robot de la marca alemana KUKA, específicamente con el modelo KR 125/2, perteneciente a la segunda generación de robots fabricados por KUKA. EI KR 125 tiene seis ejes, lo cuales le permiten moverse con 6 grados de libertad, facilitando el trabajo de fresado con una infinidad de posibilidades de orientación de la herramienta y desde cualquier punto del espacio delimitado por las restricciones de alcance del brazo. Este modelo puede mover sin problema una carga puntual de hasta $125 \mathrm{Kg}$ sobre el extremo del brazo extendido a una velocidad máxima de $2 \mathrm{~m} / \mathrm{s}$. Los movimientos pueden ser controlados mediante un mando a distancia o programados en el lenguaje nativo del robot. Este lenguaje se conoce como KUKA Robot Language (KRL), el cual es similar a Pascal, y permite programar secuencias de movimientos, declarar variables, leer sensores y trabajar con clausulas condicionales (Braumann y BrellCokcan, 2011).
Los comandos básicos en que se basan prácticamente todas las secuencias de movimientos del KR 125 son el movimiento lineal (LIN) y el punto a punto (PTP). El comando LIN permite mover la herramienta del extremo del brazo desde un punto en el espacio a otro a lo largo de una línea recta. El comando PTP permite mover la herramienta desde un punto en el espacio a otro rotando lo menos posible cada uno de sus ejes. La combinación de estos dos comandos, más la configuración del espacio de trabajo son los elementos básicos para programar en el lenguaje KRL.

\section{Métodos de corte utilizados}

Corte a lo largo de una curva base: En este método de fresado la dirección y sentido del eje de corte están determinados por la dos puntos en el espacio de trabajo. El primero se encuentra sobre una curva base, en el cual descansa el extremo de la herramienta de corte, determinando así el sentido de corte. El segundo se encuentra sobre una curva guía, el cual junto al punto base determinan la dirección del eje de corte. Para generar la trayectoria de corte es necesario subdividir ambas curvas en una sucesión de tramos rectos de igual longitud, esto genera una lista de puntos sobre cada curva. A través de esta lista de puntos se desplaza el eje de corte interpolando su ubicación actual con la siguiente, generando así un movimiento continuo sobre las curvas base y guía. Para no dañar la fresa y la amoladora, se limita la profundidad de corte a solo $3 \mathrm{~mm}$. Para logra realizar cortes con una mayor profundidad es necesario realizar varios cortes sobre la trayectoria, desplazando la curva base $3 \mathrm{~mm}$ cada vez hasta llegar a la profundidad requerida.

Corte perpendicular a una superficie: En este método la dirección y sentido del eje de corte, cuyo extremo se encuentra sobre un punto $(x, y, z)$ ubicado sobre la extensión a fresar, son idénticos al vector normal $\mathrm{N}$ relativos a esa posición sobre la superficie. La trayectoria se genera al desplazarse el eje de corte a través de los puntos distribuidos sobre la superficie. Esta distribución es determinada por una grilla de dimensiones UxV, donde cada intersección de los ejes que conforman esta grilla demarca la posición de un punto. A diferencia del método anterior cada capa de corte es generada por el extremo de la fresa, por lo tanto para poder devastar el bloque hasta su apariencia final, es necesario 
considerar la restricción de profundidad máxima de corte. Para lograr esto se debe comenzar con las capas exteriores e ir avanzando por niveles hasta llegar a la capa final.

\section{Ensambles de madera (caja y espiga)}

Paralelamente a los ejercicios realizados con estudiantes en el taller de arquitectura, se llevo a cabo un trabajo de investigación sobre la utilización de brazos robóticos en el mecanizado de ensambles de madera de caja y espiga. La posibilidad de poder cortar desde distintas direcciones permite fresar distintas partes del ensamblaje, sin necesidad de utilizar distintas máquinas para realizar todo el proceso. Al poder cambiar constantemente la orientación de la herramienta según el plano de trabajo, permite fabricar piezas de gran complejidad geométrica.
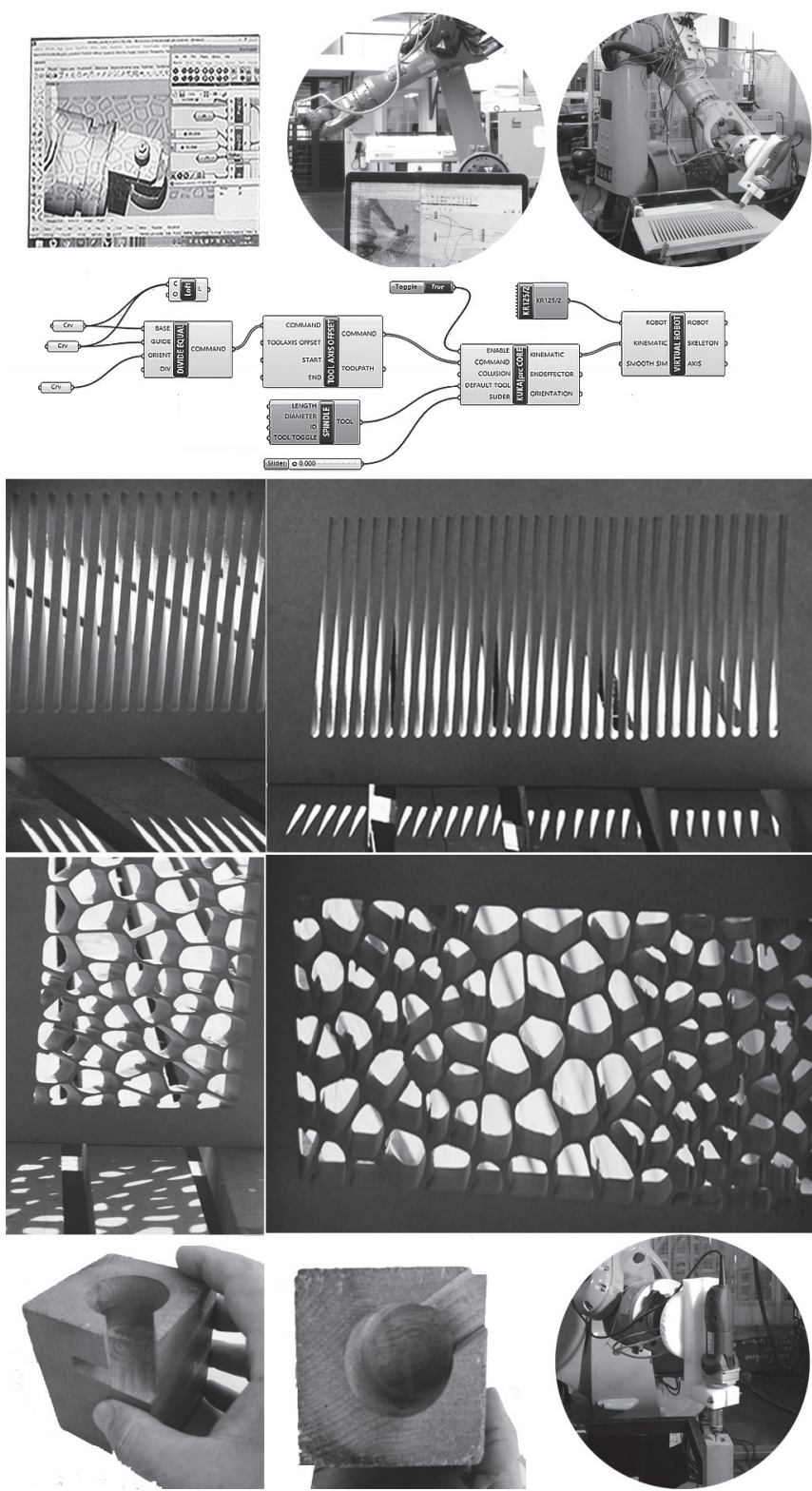

Figure 2: Componentes Variables: Celosías-Paneles y Ensambles

\section{Conclusiones}

El estudio de la automatización en construcción y diseño ha sido el tema convocante del Taller Avanzado y la investigación proyectual se basó en el estudio de las vinculaciones constructivasgeométricas por sistemas de montaje que surgen de una exploración formal pensadas para procesos CAD/CAM y la utilización de brazos Robóticos de 6 ejes. Es así como se intentó desarrollar competencias en el diseño modular y en el uso de robots industriales en la fabricación de componentes constructivos para la enseñanza de la arquitectura.

Las variadas posibilidades que ofrecen los brazos robóticos industriales en la fabricación asistida por computador, otorgan un amplio y novedoso campo de estudio para Arquitectura. Al contar con 6 grados de libertad de movimiento, estos robots permiten trabajar libremente en el espacio de trabajo. El brazo es capaz de seguir trayectorias irregulares desde cualquier dirección, permitiendo al usuario trabajar en múltiples planos de corte. Operar con estas lógicas nos propone ampliar las capacidades tecnicas constructivas en arquitectura concentrando los esfuerzos ya no solo por concebir y controlar geometrías complejas, sino por avanzar sobre una construcción coherente y con criterios racionalizados de las mismas.

Uno de los desafíos pendientes en Latinoamérica es trabajar sobre la convivencia y complementariedad de estas tecnologías de simulación e ideación post-mecánicas con las tecnologías constructivas industriales y pre-industriales heredadas. Las nuevas producciones deberán acompañar la complejidad de estos diseños, integrando técnicas y procedimientos de fabricación digital CAD/CAM para evitar que lo complejo sea solo la geometría resultante y no la ejecución de la misma. Es así como una nueva modularidad de componentes se impone proponiendo formas de ensamblajes no seriadas para la Arquitectura.

En relación con las incumbencias profesionales, se visualiza, con estas tecnologías, mejores posibilidades de autogestión de emprendimientos en equipos interdisciplinarios, propios de una nueva generación de profesionales que no ven suficiente quedar a la espera de un mecenas para desarrollar la totalidad de sus proyectos. Es así como es posible y necesario enriquecer progresivamente nuestras competencias académicas incorporando saberes, problemas e instrumentos propios de los nuevos entornos tecnológicos. Esta incorporación incipiente de la automatización en construcción y diseño para la enseñanza de la Arquitectura en un Taller Avanzado, mediante el estudio de las vinculaciones constructivas-geométricas por sistemas de montaje con brazos Robóticos de 6 ejes, continuará explorando sobre las posibilidades de una transformación o complementariedad en los procesos de fabricación y construcción utilizados en arquitectura. 

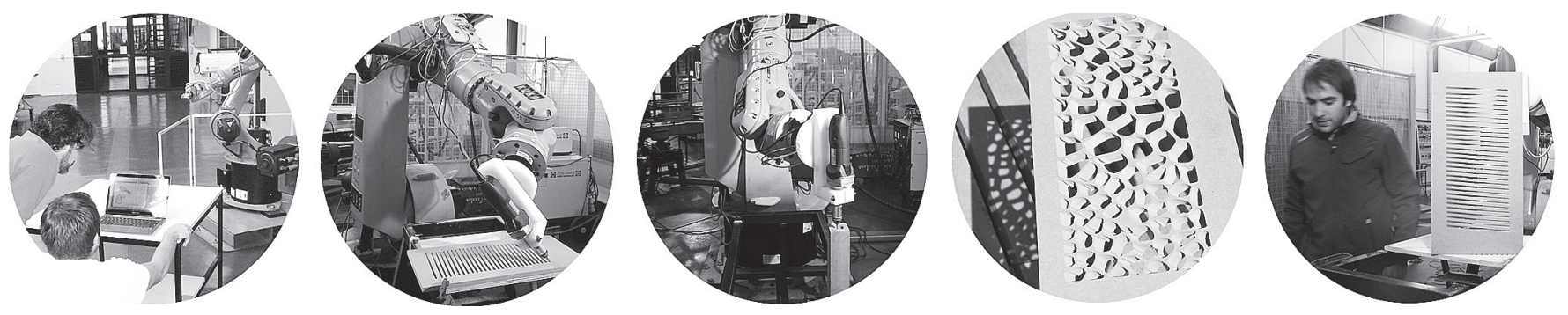

Figure 3: Automatización en Diseño y Construcción: KUKA kr125

\section{Agradecimientos}

Agradecemos especialmente a Pablo Escalona, Maximiano López y Mauricio Solís integrantes del staff del Centro Integrado de Manufactura y Automatización (CIMA), al director del Departamento de Ingeniería Mecánica de la UTFSM Prof. Dr. Ing. Eugenio González Vergara, a la directora del Departamento de Arquitectura de la UTFSM Prof. PhD Nina Hormazábal, a Johannes Braumann de la "Association for Robots in Architecture", a los alumnos del taller avanzado de diseño arquitectónico ACD_2013 (Automatización en Construcción y Diseño) y a Pablo Encina del Laboratorio de Materialidad (LABOMAT) del Departamento de Arquitectura de la UTFSM.

El Taller Avanzado sobre Automatización en Diseño y Construcción, se dictó el primer semestre de 2013 en la carrera de Arquitectura de la Universidad Técnica Federico Santa María. Los Profesores a Cargo han sido: Arq. Luis Felípe González y Arq. Cristián Clavo. El Investigador Dr. Mauro Chiarella participó del Taller como Profesor externo invitado.

\section{Referencias}

Braumann, J; Brell-Cokcan, S. (2011) Parametric Robot Control: Integrated CAD/CAM for Architectural Design, ACADIA 11: Integration through Computation [Proceedings of the 31st Annual Conference] 13-16 October, 2011, pp. 242-251

Chiarella, M. (2004). Geometry and Architecture: NURBS, Design and Construction. Journal of Mathematics \& Design. The International Mathematics \& Design Association; Buenos Aires; vol. 4 p. 135-139

Kohler, M; Gramazio, F. (2008). Digital Materiality in Architecture. Baden: Lars Müller Publishers.

KUKA Roboter GmbH (2005). Manual Software KR C2 / KR C3, Programación por el experto, KUKA System Software (KSS), Release 5.2. Edición: 12.09.2005. Versión: 01

Association for Robots in Architecture (http://www.robotsinarchitecture.org/).

Automatización en Diseño y Construcción (http://adc2013.wordpress.com/category/novedades/) 